\title{
Funding Global Public Goods: The Dark Side of Multilateralism *
}

\author{
Patrick Bayer \\ University of Mannheim \\ Johannes Urpelainen ${ }^{\dagger}$ \\ Columbia University
}

11 July 2012

\begin{abstract}
The funding of global public goods, such as climate mitigation and biodiversity conservation, presents a complex strategic problem. Potential recipients demand side payments for implementing projects that furnish global public goods, and donors can cooperate to provide the funding. We offer a game-theoretic analysis of this problem. In our model, a recipient demands funding for a project. Donors can form a multilateral funding program to jointly fund the project. If no program is formed, bilateral funding remains a possibility. We find that donors rely on multilateralism if their preferences are relatively symmetric and domestic political constraints on funding are lax. In this case, the recipient secures large rents from project implementation. Thus, even donors with strong interests in global public good provision may oppose institutional arrangements that promote multilateral funding. For example, even environmentally aware donor countries have incentives to oppose multilateral climate finance. These incentives have played an important role in multilateral negotiations on climate finance, especially in Cancun (2010) and Durban (2011).
\end{abstract}

\footnotetext{
${ }^{*}$ This paper was written during a research stay funded by an ERP fellowship of the Studienstiftung des deutschen Volkes. Patrick Bayer gratefully acknowledges this generous funding and is thankful for the hospitality of Columbia University. We thank Scott Barrett, Joe Brown, and Chris Marcoux for useful comments on a previous draft.

${ }^{\dagger}$ Corresponding author. Assistant Professor, Department of Political Science, Columbia University. 420 West 118 th Street, 712 International Affairs Building. New York, NY 10027, USA. Phone: +1-734-757-0161. Email: ju2178@ columbia.edu.
} 


\section{Introduction}

Many national policies produce global public goods, such as climate mitigation and biodiversity conservation (Anand, 2004; Goeschl and Swanson, 2002; Victor, 2011). However, unless these national policies produce domestic benefits that exceed domestic costs, national governments have few incentives to act. Without international cooperation, many valuable global public goods remain in short supply. For example, this problem is repeatedly encountered in negotiations on finance for climate mitigation in developing countries (Stewart, Kingsbury, and Rudyk, 2009). The global environmental benefits of decarbonizing industrial growth in China and other rapidly growing countries are massive, but a large share of those benefits accrue to people outside these countries. Without external assistance, climate mitigation projects with excellent global cost-benefit ratios are thus not implemented.

Given this reality, both scholars and policymakers have recently emphasized the importance of international arrangements for the funding of global public goods (Barrett, 2007; Kaul, Grunberg, and Stern, 1999; Sandler, 2004). The central finding of this literature is that, except for unusual circumstances, inducing donors to participate in the funding of global public goods is difficult (Barrett, 2001; Hoel and Schneider, 1997; Sandler, 2004). Possible donors want others to fund the global public goods, so free riding presents an impediment to effective funding arrangements.

Though valuable, these approaches neglect the strategic effects of multilateral funding programs. For instance, China's demands for climate finance in exchange for project implementation are strategic decisions that depend on the expected donor response. These demands may differ depending on the availability of bilateral and multilateral funding. If donors can form a multilateral funding program, how does this influence their expected payoff from global public goods and the probability of successful project implementation?

Recent decisions at the 17th session of the Conference of the Parties in Durban stress the importance of climate finance. In their draft decision on the Green Climate Fund, the parties agreed that this "Fund will play a key role in channeling new, additional, adequate and predictable financial resource to developing countries and will catalyse climate finance, both public and private, at the international and national levels". ${ }^{1}$ In a similar vein, the Ad-hoc Working Group on Long-term Cooperative Action under the Convention

\footnotetext{
${ }^{1}$ See http://unfccc.int/files/meetings/durban_nov_2011/decisions/application/pdf/cop17_ gcf . pdf. Accessed December 20, 2011.
} 
reiterates the claim "that a wide variety of new and existing sources of financing, public and private, bilateral and multilateral" is needed to reduce emissions from deforestation and forest degradation in developing countries (REDD plus). ${ }^{2}$

We analyze a game-theoretic model focused on the problem of funding global public goods. In the model, a recipient country can demand funding for implementing a project that produces global public goods. Donors must then decide on participation in a multilateral funding program. If they participate, they bargain over burden sharing. If they do not participate, bilateral funding remains a possibility. To incorporate domestic political constraints, we assume that each donor is, with some probability, not in a position to fund global public goods.

Our primary finding can be characterized as the dark side of multilateralism, from the perspective of donors. When donors and recipients bargain over project implementation, they must somehow distribute the rents from the project. While our model cannot address the normative question of how these rents ought to be distributed, the model does shed light on what may be expected. If the donors' preferences concerning the global public good are approximately symmetric and political constraints are lax, the recipient demands a large amount of funding for project implementation. Since the recipient believes that the donors will be in a position to form a multilateral funding program, the recipient can extract large rents from the donors. The multilateral funding program internalizes the global benefits of global public goods, so the recipient can demand a much higher price for project implementation. Thus, donors' efforts to fund global public goods reduce their own payoff.

A direct corollary to this finding is that donors have incentives to credibly commit to not forming multilateral funding programs. Donor-recipient bargaining endows donors with incentives to refrain from collective action to provide global public goods. For example, this finding is highly relevant to present difficulties in the formation of climate finance institutions. Industrialized countries have incentives to oppose multilateral climate finance even if they have a strong interest in climate protection.

A particularly interesting example of the strategic logic of multilateral funding is Ecuador's offer to leave oil under the Yasuni National Park untapped. In September 2007, Ecuador's President Rafael Correa proposed that the country leave oil below the national park - a UNESCO world heritage site and a biodiver-

\footnotetext{
${ }^{2}$ See http://unfccc. int/resource/docs/2011/awglca14/eng/crp37.pdf. Accessed December 20, 2011.
} 
sity hotspot - untapped in exchange for a side payment from foreign donors. ${ }^{3}$ Consistent with our model, in August 2010 the United Nations Development Program and Ecuador indeed formed a trust fund to provide Ecuador with US\$ 3,600 million in exchange for leaving the oil underground, and "[c]ountries including Germany, the Netherlands, Norway, Italy and the US have expressed interest in contributing." ${ }^{4}$ In exchange for leaving one-fifth of the country's oil reserves untapped, and thus protecting a highly valuable diversity hotspot, Ecuador received a large side payment from a multilateral initiative formed by donor countries. ${ }^{5}$

Moreover, we find that domestic political constraints in donor countries may reduce the recipient's ability to demand a large side payment. If some donors are highly constrained while others are not, the recipient's optimal strategy is to demand amounts of funding that the less constrained donors are actually willing to provide. Thus, a donor with stringent political constraints is in a position to free ride on other donors' contributions. Even highly interested governments may see few demands for bilateral funding if their domestic political institutions impose stringent constraints on executive authority. This finding is broadly consistent with the "paradox of the weak" that Schelling (1960) and others (Milner, 1997; Putnam, 1988) have emphasized, though the strategic logic is quite different.

One broader theoretical implication of the analysis is the surprising effect of collective action on different countries' payoffs. Constraints on international collective action can produce large benefits for groups of states engaged in bargaining with other groups of states. In our model, for example, domestic political constraints in donor countries reduce recipients' ability to extract rents. The policy implications are also notable. Even donors with a strong interest in climate cooperation, such as the European Union, have incentives to oppose effective multilateral institutions for climate finance. According to our model, one way out of this trap is the creation of multilateral finance arrangements that impose a firm upper bound on funding.

\footnotetext{
3“Ecuador Seeks Oil Compensation.” BBC September 21, 2007. See http://news.bbc.co.uk/2/hi/americas/ 7000345 . stm. Accessed August 22, 2011.

4"Ecuador Pledges No Oil Drilling in Amazon Reserve." BBC August 3, 2010. See http://www.bbc.co.uk/news/ world-latin-america-10861415. Accessed August 22, 2011.

${ }^{5}$ Harstad (2011) presents a game-theoretic model of climate policy in deposit markets for fossil fuels.
} 


\section{Global Public Goods}

By global public goods, we refer to the implementation of projects that produce non-rival and non-excludable benefits for large groups of people in different countries across the globe (Anand, 2004, 216). Global public goods are distinct from national or regional public goods because the group of beneficiaries is more evenly spread across the world (Barrett, 2007; Sandler, 2004). Potential examples of global public goods include biodiversity conservation (Goeschl and Swanson, 2002), climate mitigation (Fischer and Newell, 2008), scientific research (Stiglitz and Wallsten, 1999), and vaccination campaigns (Barrett, 2007). Our focus here is on funding the provision of global public goods in specific (recipient) countries by foreign (donor) countries.

Global public goods have drawn the attention of scholars and policymakers at least since the end of the Cold War (Anand, 2004; Kapur, 2002; Kaul, Grunberg, and Stern, 1999). Economic analyses of global public goods emphasize the importance of aggregation technology, the presence of a leading state capable of coordinating the behavior of others, and excludable national side benefits (Barrett, 2007; Sandler, 2004; Sandler and Hartley, 2001). This literature focuses on issues of collective action among sovereign states. Since cooperation cannot be enforced externally, through the formation of an omnipotent world government, cooperative arrangements must be "self-enforcing" (Barrett, 1994; Carraro and Siniscalco, 1993). According to these models, forming self-enforcing agreements with expansive membership and stringent policies is difficult because each state has an incentive to free ride. Even if the treaty itself is enforceable, states may choose to remain outsiders. Therefore, non-participatory free riding is an obstacle to global public good provision.

However, this literature does not account for the specific problems of funding projects in recipient countries. Most models within this literature simply ignore these issues, and instead focus on reciprocal policy adjustments. Even models that do consider North-South side payments (Barrett, 2001; Hoel and Schneider, 1997) ignore bargaining problems and the role of domestic political constraints in donor countries. Although early work by Putnam (1988) and Iida (1993) on two-level games highlights the importance of domestic constraints for bargaining outcomes, it does not examine problems of international public good provision.

Theories of foreign aid offer another set of insights into the funding of global public goods. Some authors focus on bargaining among donors, emphasizing the importance of voting rules and coalitional 
power in multilateral organizations (Nielson and Tierney, 2003). Others argue that multilateral foreign aid can help governments convince their audiences that the foreign aid is used to promote good causes (Rodrik, 1995; Milner, 2006). Yet others emphasize the donor's problem of providing the recipient with incentives to adjust policies in exchange for foreign aid (Bueno de Mesquita and Smith, 2007; Drazen, 2002). And, yet others examine the Samaritan's dilemma: how can donors commit to not helping recipients who fail to improve the lot of the poor (Pedersen, 2001; Hagen, 2006)?

This research is useful insofar as it sheds light on distributional conflict between donors and recipients, as well as highlights the advantages and disadvantages of bilateralism and multilateralism from a donor perspective. However, most of the foreign aid literature does not consider the implications of global public goods. With few exceptions, such as Torsvik (2005), conventional models have one donor and one recipient. ${ }^{6}$

Additionally, the role of domestic politics in donor countries is frequently downplayed in the foreign literature. One exception is Bueno de Mesquita and Smith (2007). They illuminate a donor's willingness to purchase policy concessions that produce national public goods for its citizens. Another exception is Chong and Gradstein (2008). They find that wealth and good governance in donor countries increases the supply of foreign aid. However, neither Bueno de Mesquita and Smith (2007) nor Chong and Gradstein (2008) examine donor-recipient bargaining on global public good provision in view of domestic politics. Lancaster (2007) overviews the domestic politics of foreign aid in several donor countries, but she does not present a model thereof.

Against this backdrop, we examine a model that incorporates donor collective action, domestic political constraints on funding, and the bargaining relationship between recipients and donors. Our model characterizes donors' incentives to form multilateral programs that facilitate the funding of global public goods, recipients' strategies concerning project implementation, and the effects of domestic political constraints.

\footnotetext{
${ }^{6}$ Torsvik (2005) does not analyze donor-recipient bargaining.
} 


\section{Model}

We explore the strategic dimensions of donor-recipient interactions in the funding of global public goods. ${ }^{7}$ The players are a recipient and two donors, denoted by $A, B .{ }^{8}$ In the main model, the recipient can propose to the donors that a project be implemented. ${ }^{9}$ The proposal is associated with a demand for a side payment, denoted by $t \in[0, \infty)$.

As we develop the model, we pay particular attention to two issues. ${ }^{10}$ First, how does the fact that donors can form a multilateral funding program (MFP) to fund global public goods influence the recipient's demand for a side payment and the donors' response? Second, how do domestic political constraints in the donor countries influence equilibrium behavior and outcomes?

The sequence of the moves can be described as follows:

1. The recipient issues a demand for the side payment $t$.

2. Nature draws and publicly reveals a type for each donor $i=A, B$.

3. Each donor $i=A, B$ decides on participating in the MFP, $M_{i} \in\{0,1\}$.

4. Funding decisions are made as follows:

(a) If at least one donor rejects the MFP, then each donor $i=A, B$ accepts or rejects the recipient's project, $F_{i} \in\{0,1\}$.

(b) If both donors participate in the MFP, then the donors play a Nash Bargaining Game over funding the recipient's project.

For simplicity, the recipient holds the initiative. ${ }^{11}$ This modeling choice is grounded in Victor's (2011)

\footnotetext{
${ }^{7}$ Our model focuses on interactions between countries that can clearly be identified as donors who provide funding and recipients who obtain funding. However, a particular country can be a donor in one project while being a recipient in another project. In the Global Environment Facility, China plays such a double role (see http://www. worldbank. org/en/country/china/ projects/all?qterm=GEF and http://www.thegef.org/gef/trust_funds).

${ }^{8}$ Our results do not depend on the exact number of donors. As long as more than one exists, the strategic incentives remain intact.

${ }^{9}$ The model can also be applied in the case of multiple recipients. In this case, the donors must decide on providing funding for multiple projects in different countries. This gives the donors more bargaining power if the projects are strategic substitutes for each other. If they are not related, however, the funding of each can be analyzed as a separate strategic game.

${ }^{10}$ To simplify, we omit some previously recognized benefits of multilateral funding, such as economies of scale (Abbott and Snidal, 1998) and signaling good intent to domestic audiences (Milner, 2006).

${ }^{11}$ We verify in a mathematical extension that our results hold even if the donors can credibly commit to rejecting very aggressive demands.
} 
approach to funding global public goods. Governments in recipient countries are best positioned to identify projects that lend themselves, politically and economically, to foreign funding. The recipients can then propose these projects to putative donors. For example, South Africa could signal its interest in replacing planned coal power plants with wind power parks. ${ }^{12}$

To assume there is only one recipient country is a strong simplification. However, even with multiple recipients competing for donor funding, recipients would have incentives to inflate their offers to extract additional revenue from multilateral funding initiatives. However, the potential bargaining leverage that recipients have decreases in the number of recipient countries. This ultimately favors MFP formation.

Moreover, our approach is realistic in that recipients could join forces and form a coalition; if they speak with one voice at the bargaining table, multiple recipients would behave as if there was only one recipient country. In the context of climate change, it is not uncommon that groups such as the Alliance of Small Island States ${ }^{13}$ or the developing countries' group $\mathrm{G} 77^{14}$ bargain jointly. At the 15 th Conference of the Parties in Copenhagen, Denmark, in 2009 the "G77-China bloc [which] speaks for developing countries in the climate change negotiation process"15 jointly suspended cooperation. Given this, our model appears warranted.

The formation of an MFP for funding admits multiple plausible interpretations. If the recipient's offer is interpreted as a general guideline for project implementation in the future, such as a rule for burden sharing, then MFP formation could be interpreted as the formation of a large institution, such as a financial organization. For example, developing countries could propose a resource allocation rule for climate finance in the coming years.

If the recipient's offer pertains to a specific project, then MFP formation can be interpreted as a smaller multilateral initiative. For example, a developing country could propose that donors fund an increase in the use of natural gas instead of coal in power generation. The donors could then bargain over securing funding for the project within the confines of the World Bank or the Global Environmental Facility.

\footnotetext{
${ }^{12}$ For the opposite assumption of donor initiative, see Barrett (2001). This approach is suitable for side payments used to induce recipients to join a treaty, because such side payments can be thought of as an accession bonus. However, the approach is not suitable when individual recipients can identify and propose projects.

${ }^{13}$ See http://aosis.info/aomembers/. Accessed May 28, 2012.

${ }^{14}$ See http: / /www.g77.org/doc/members.html. Accessed May 28, 2012.

15 "Copenhagen Climate Summit Negotiations 'Suspended'.”, BBC December 14, 2009. See http://news .bbc. co.uk/ 2/hi/8411898.stm. Accessed May 28, 2012.
} 
If donor bargaining within the MFP fails, so that "disagreement" (Nash, 1950) prevails, we assume the project is not implemented. As we show in the appendix, this seemingly strong assumption is innocuous: in equilibrium play, bargaining failure within the MFP cannot be followed by a donor's unilateral decision to fund the project.

The recipient's payoff is simply the side payment $t$ less the cost of project implementation, $C>0$. The $\operatorname{cost} C$ depends on the net national loss from project implementation without external funding. For example, in the case of biodiversity conservation the value of $C$ would increase with the commercial value of clearing rainforest for agriculture and decrease with the direct benefits of conserving that rainforest, such as food and firewood for people living in the area.

The donor's payoff depends on its Bayesian type. This Bayesian type captures the effect of domestic political constraints. In the main text, we adopt a particularly simple approach: with some probability $p_{i} \in$ $[0,1]$, the donor is "constrained", and thus categorically unable to fund the project. With the complementary probability $1-p_{i}$, the donor is "unconstrained", and thus interested in funding the project if the benefits exceed the costs. These probabilities are, for simplicity, independent of each other across the two donor countries. The constrained type could, for example, be thought of as a donor country with a domestic veto player that has no interest in global public goods (Tsebelis, 2002). Such veto players are, for example, influential in climate policy in the United States (Agrawala and Andresen, 1999). The probability $p_{i}$ captures the possibility that these veto players manage to prevent budgetary allocations for funding the provision of global public goods in the recipient country.

The donors' types are not revealed to the recipient at the initial demand stage. The recipient cannot perfectly anticipate domestic political constraints in donor countries, so it must formulate the funding proposal under uncertainty. This assumption is plausible for two reasons. First, the delay between a funding proposal and political decisions by donors can be years. Thus, even if the recipient is aware of domestic political constraints at the time the proposal is formed, it cannot perfectly foresee the future. Second, governments in donor countries may have private information about the possibility of forming a political coalition in support of the funding proposal.

For simplicity, we assume in the main text that the donors' types are publicly revealed before they move. This assumption allows us to focus on how uncertainty influences the recipient's behavior without 
creating a complex bargaining model under incomplete information between donors. The assumption is also rather plausible in that, as soon as the exact nature of the funding proposal is revealed, political debate in donor countries begins and new information is available. In an extension, we then show that incomplete information between the donors does not compromise our main findings.

Although our model is static in that learning about political constraints is not explicitly modeled, the assumption that donor types are publicly revealed can be perceived as the result of learning from iterative play. ${ }^{16}$ If our model was played repeatedly, donors would learn about domestic opposition, i.e., about their types. Over time, this learning scenario yields identical results as our model with the assumption that donor types are publicly revealed.

For the unconstrained type, the cost of a side payment $t$ is simply $-t$. If the project is not implemented, donor $i$ suffers damage worth $D^{i}>0$. This damage could reflect contagious diseases, loss of biodiversity, or a worsening international security environment. If the project is implemented, this environmental damage is avoided. Thus, the global value of the project is $D^{A}+D^{B}-C$, assumed to be strictly positive. Importantly, this formulation captures the assumption of global public goods: the project benefits both donors, but neither internalizes the total global benefits. In our model, however, this does not mean that project implementation is an easy success: domestic political constraints may result in failure, and our equilibrium analysis characterizes the probability of such failure. Without loss of generality, suppose $D^{A}>D^{B}$ so that donor $A$ is more enthusiastic about global public goods. Suppose also $D^{B}>C$ so that both donors are interested in funding.

For generality, the public good value $D^{i}$ is modeled separately from the constraint probability $p_{i}$. Based on previous empirical research on foreign aid, this assumption is substantively plausible. For example, as Hicks et al. $(2008,9)$ write, even if donor countries value environmental protection in recipient countries, providing environmental aid "still costs an enormous amount of money ... [and] funding increases have a long series of checks, balances, budgets, committees, agencies, and other political institutional hurdles to pass." Even the most environmentally aware industrialized countries have domestic groups who oppose foreign aid. Thus, controlling for the observable ecological vulnerability and preferences $D^{i}$ of a donor country (Sprinz and Vaahtoranta, 1994), domestic political constraints (Tsebelis, 2002) may intervene. This

\footnotetext{
${ }^{16}$ Our static model offers new insights into the strategic nature of multilateral global public good funding, and a more fully developed dynamic learning model may be the next step for future research.
} 
is what the constraint probability $p_{i}$ captures.

Consider finally the funding of the project. Suppose, first, that no MFP has been formed because $M_{i}=0$ for at least one of $i=A, B$. In this case, the project is not funded unless at least one donor $i=A, B$ accepts the recipient's offer. If only one donor $i$ accepts, it pays the side payment $t$ and the project is implemented to the benefit of both donors. If both donors accept, the more interested donor $A$ funds it. ${ }^{17}$ In the complete information version of the game, it also turns out that on the equilibrium path of play, donor $B$, who is less enthusiastic about climate change mitigation, never has any incentive to fund the project.

Suppose now that an MFP is formed, as $M_{A}=M_{B}=1$. In this case, the two donors play a conventional Nash Bargaining Game. In this game, the disagreement payoffs are $-D^{A},-D^{B}$ : if funding fails, then each donor suffers from pollution. ${ }^{18}$ Cooperation is characterized as project funding, and it produces a value of $-t$, as the donors avoid global damage worth $D^{A}+D^{B}$ but must pay the side payment $t$. As usual, the game is solved for the Nash Bargaining Solution. The technical details can be found in the appendix. According to this solution, unless the project's cost exceeds the global benefit, each donor receives $\frac{1}{2}$ of the added value of funding the project. Thus, if the project is funded, the payoff to donor $i$ is

$$
-D^{i}+\frac{1}{2}\left(-t+D^{A}+D^{B}\right)
$$

The first term is donor $i$ 's disagreement point and the second term is the added value of funding the project.

\section{Equilibrium}

To build intuition, we analyze the game first under complete information. Drawing on backward induction, we apply subgame-perfect Nash equilibrium as solution concept. For this, we first solve the subgame between donors $A$ and $B$ for optimal MFP formation and funding decisions. We then extend our model to an incomplete information game in which the recipient country is uncertain about player types in donor states $A$ and $B$. To solve this game, we use the perfect Bayesian Nash equilibrium solution concept.

\footnotetext{
${ }^{17}$ This is an equilibrium outcome of the game. The only reason we need a tiebreaker rule is to ensure uniqueness.

${ }^{18}$ As noted above, in the appendix we show that this would be the unique equilibrium outcome on the path of play even if we allow individual donors to offer unilateral funding upon bargaining failure. Specifically, we show that neither donor would do so.
} 


\subsection{Complete Information}

In the complete information game, both donors are of the unconstrained type, $p_{A}=p_{B}=0$. Without this assumption, only one donor could fund the project. This would prevent an analysis of donor collective action and result in the trivial conclusion that the recipient demands funding from the only possible donor.

Solving the game backwards, we need first to find a solution to what we call the "MFP formation subgame". In this subgame, both donors $A$ and $B$ maximize individual utility conditional on the optimal side payment $t^{*}$ demanded by the recipient.

Suppose first that no MFP forms. Given this, both donors $A$ and $B$ can only fund the climate project unilaterally. As the benefits for both donors $i$ are the avoided climate damages $D^{i}$, the maximal side payment offers the two donors would accept are $t^{*}=D^{A}$ and $t^{*}=D^{B}$, respectively. Without loss of generality we assumed that country $A$ is more vulnerable to climate change, i.e., $D^{A}>D^{B}$ holds. Consequently, donor $A$ is the one who reaps higher benefits from project implementation compared to country $B$. Therefore, the more ambitious donor $A$ funds the project.

Imagine now that donors $A$ and $B$ decide to form an MFP. For both donors to participate, their payoffs from MFP formation must both be better than the payoffs from the alternative options of unilateral project funding or no project implementation at all. For donor $A$ the payoff from MFP formation is given as $\frac{1}{2}\left(D^{B}-D^{A}-t^{*}\right)$ from equation (1), which is only larger than the payoff $-t^{*}$ from unilateral funding if $D^{A}-D^{B} \leq t^{*}$. Applying a similar logic, we find that forming an MFP gives higher returns for $A$ compared to no project implementation at all if $t^{*} \leq D^{A}+D^{B}$. In essence, for donor $A$ MFP participation is utility maximizing if the recipient's side payment request is such that it falls in between the lower and upper boundaries $D^{A}-D^{B} \leq t^{*} \leq D^{A}+D^{B}$.

Since country $B$ is the less ambitious donor, it does not fund the project unilaterally. Therefore, its participation decision depends on whether donor $A$ provides the public good unilaterally or if MFP formation fails. Assume first that $t^{*} \leq D^{A}$ holds and the side payment would be sufficiently small, so that donor $A$ would fund the project unilaterally. Given this, MFP formation is only rational for $B$ if the participation payoff $\frac{1}{2}\left(D^{A}-D^{B}-t^{*}\right)$ is larger than the zero payoff from free-riding. This is true as long as the side payment $t^{*} \leq D^{A}-D^{B}$ is so small that the utility transfer for $B$ from within the MFP outweighs the costs of the side payment. Alternatively, let us suppose that $D^{A}<t^{*}$, so that unilateral funding by $A$ and, 
therefore, free-riding by $B$ is not an option. If $t^{*}$ is high enough, not participating in the MFP by $B$ would sacrifice project implementation. This is why $B$ participates in an MFP if $D^{A}<t^{*} \leq D^{A}+D^{B}$. With these mutual best-responses it is easy to show that an MFP either forms if the side payment $t^{*}$ falls within the interval $\left(D^{A}, D^{A}+D^{B}\right]$ or if $t^{*}=D^{A}-D^{B}$. These two offers are the equilibrium points in the MFP formation subgame.

With a rational recipient that maximizes utility, higher side payments translate into higher utility. Recall that $D^{B}>C$. Therefore, the recipient offers $t^{*}=D^{A}+D^{B}$. Thus, we have the following subgame perfect Nash equilibrium for the complete information game: the recipient makes a maximally extractive side payment offer $t^{*}=D^{A}+D^{B}$, an MFP forms, and the project is funded.

This result shows that MFP formation benefits the recipient. Since the MFP internalizes the global benefits of the project, it accepts more aggressive demands than any individual donor would. The recipient thus proposes a large demand, $t^{*}=D^{A}+D^{B}$, and the donors form an MFP to fund it. Under complete information, the recipient knows that increasing the offer beyond $A$ 's upper limit for implementing the project unilaterally induces $B$ to participate in the MFP. Since $B$ rightfully fears non-implementation if $t^{*}$ exceeds $A$ 's valuation of the project $D^{A}$, it is rational for $B$ to form an MFP. As the MFP pools the donors' financial resources, the recipient can extract more rents than under bilateral funding.

\subsection{Incomplete Information}

Under incomplete information, we rely on the perfect Bayesian equilibrium. For each donor, the constrained type never participates in the MFP or funds the project. Since the donors' types are revealed prior to their decisions, we need not characterize posterior beliefs. Given the recipient's offer, and the revealed donor types, each donor decides on MFP formation and funding in a rational fashion.

The MFP formation subgame remains unchanged. Incomplete information is a problem for the recipient country, whereas donors can bargain under complete information if the MFP forms. ${ }^{19}$ Similarly, if the MFP fails to form, the decisions to fund the project remain unchanged. Finally, this is true of the decision to form an MFP, with the exception that if one of the donors is constrained, then an unconstrained donor's participation decision is completely irrelevant.

\footnotetext{
${ }^{19}$ In the mathematical appendix, we show that the same equilibria are sustained when donor countries are uncertain about their own domestic constraints.
} 
But how should the recipient select the side payment demand under incomplete information? We find that in equilibrium, the recipient country can either demand a small side payment $t^{*}=D^{B}$, an intermediate one $t^{*}=D^{A}$, or a high one $t^{*}=D^{A}+D^{B}$.

Given her prior beliefs about $p_{A}$ and $p_{B}$, the recipient selects the offer to maximize its expected utility. The expected utilities are the following:

$$
\begin{aligned}
D^{B}\left(1-p_{A}\right)\left(1-p_{B}\right)+D^{B}\left(1-p_{B}\right) p_{A}+D^{B}\left(1-p_{A}\right) p_{B} & \text { if } t=D^{B} \\
D^{A}\left(1-p_{A}\right)\left(1-p_{B}\right)+D^{A}\left(1-p_{A}\right) p_{B} & \text { if } t=D^{A} \\
\left(D^{A}+D^{B}\right)\left(1-p_{A}\right)\left(1-p_{B}\right) & \text { if } t=D^{A}+D^{B}
\end{aligned}
$$

These expected utilities have natural interpretations. If the recipient demands $t=D^{B}$, then either donor is willing to fund it as long as political constraints do not exist. Therefore, the probability of securing funding is high while the value of this funding is low. If the recipient demands, $t=D^{A}$, the probability is lower because only donor $A$ could potentially fund. If the recipient demands, $t=D^{A}+D^{B}$, then the probability is even lower because both recipients must agree for funding. In sum, the recipient faces a risk-return tradeoff.

We can now derive the following three conditions:

$$
\begin{gathered}
\frac{D^{A}}{D^{B}} \leq \frac{1-p_{B}}{p_{B}} \quad \text { if this holds, then } t^{*}=D^{A}+D^{B} \text { not } t^{*}=D^{A} \\
\frac{\left(1-p_{B}\right) p_{A}+\left(1-p_{A}\right) p_{B}}{\left(1-p_{A}\right)\left(1-p_{B}\right)} \leq \frac{D^{A}}{D^{B}} \quad \text { if this holds, then } t^{*}=D^{A}+D^{B} \operatorname{not} t^{*}=D^{B} \\
\frac{\left(1-p_{B}\right) p_{A}+\left(1-p_{A}\right) p_{B}}{\left(1-p_{A}\right)} \leq \frac{D^{A}}{D^{B}} \quad \text { if this holds, then } t^{*}=D^{A} \operatorname{not} t^{*}=D^{B}
\end{gathered}
$$

The first condition, equation (3a), states that if donor $A$ 's valuation is too large relative to donor $B$ 's, then the recipient never has an incentive to induce MFP formation through an aggressive demand. If donor $B$ 's willingness to fund is low compared to the odds that donor $B$ is constrained, MFP formation is a risky strategy. The punchline is that if donor $A$ is probably willing and able to offer generous funding, then gambling on donor $B$ 's willingness is not an optimal strategy for the recipient.

The second condition in equation ( $3 \mathrm{~b}$ ) compares strategies $t^{*}=D^{A}+D^{B}$ and $t^{*}=D^{B}$. As we show 
below, this condition turns out to be rather irrelevant. If the large demand $D^{A}+D^{B}$ results in a higher expected utility than $D^{B}$, we find, then $D^{A}$ also does so. Again, the intuition is that if gambling on $A$ 's willingness to fund is optimal, it must also be optimal without MFP formation.

Condition (3c) compares the two possible strategies for bilateral funding. It shows that if donor $A$ 's willingness to fund is higher than donor $B$ 's willingness to do so, as captured in the $\frac{D^{A}}{D^{B}}$ ratio, then gambling on donor $A$ is optimal. The offer $D^{B}$ is a safer bet because even if donor $B$ is constrained, donor $A$ will fund the project with probability $1-p_{A}$. But if donor $A$ 's willingness to pay is much larger, gambling on donor $A$ is optimal.

In conclusion, gambling on institutionalization and making most extractive offers $t^{*}=D^{A}+D^{B}$ is only optimal if both countries are unconstrained domestically and similar with respect to the damage they suffer due to project failure $D^{A}$ and $D^{B}$. If this is not the case, the recipient either plays safe by offering $D^{B}$, so that either donor is willing to fund unless politically constrained, or offers $D^{A}$ so that funding depends on donor A's domestic political constraints.

The extractive potential of the recipient decreases with uncertainty. Under incomplete information, the most extractive side payment request $t^{*}=D^{A}+D^{B}$ is only an equilibrium strategy if the probabilities of domestic opposition are low. Increasing uncertainty about domestic constraints in the donor countries induces the recipient to water down its demand.

\section{Implications}

We now summarize the central implications of our incomplete information model. To do this, we present three propositions that together offer a complete characterization of equilibrium. They also provide testable comparative statics. We first present the propositions, then offer a graphical illustration, and finally discuss the implications and some concrete examples of these results.

To begin with, we characterize the conditions under which the recipient plays safe by demanding a small side payment, $t^{*}=D^{B}$.

Proposition 1 (Small side payment). If $\frac{D^{A}}{D^{B}}<\frac{\left(1-p_{B}\right) p_{A}+\left(1-p_{A}\right) p_{B}}{1-p_{A}}$, the recipient demands $t^{*}=D^{B}$. No MFP is formed, and the project is funded unless both donors are politically constrained. 
Suppose the ratio of $D^{A}$ to $D^{B}$, or the ratio of donor $A$ 's valuation to donor $B$ 's valuation, is low while the probability that donor $A$ is constrained, $p_{A}$, is high. In this case, the recipient prefers the safe but low side payment, $D^{B}$, to the higher but risky side payment, $D^{A}$. In the appendix, we show that in this case, the highest possible side payment, $t^{*}=D^{A}+D^{B}$, also cannot be optimal. This is quite intuitive: the donors cannot form an MFP if donor $A$ is constrained. Thus, if the donor is probably constrained, then MFP formation will probably fail.

What about a somewhat higher side payment, $t^{*}=D^{A}$ ? This strategy is somewhat more dangerous than the previous one, because only donor $A$ is potentially interested in funding this. Donor $B$ would never unilaterally accept this demand because it is too costly.

Proposition 2 (Intermediate side payment). Suppose $\frac{D^{A}}{D^{B}}>\frac{\left(1-p_{B}\right) p_{A}+\left(1-p_{A}\right) p_{B}}{1-p_{A}}$. If $p_{B}>\frac{1}{2}$ or $p_{A}>$ $\frac{1-p_{B}\left(1-p_{B}\right)}{1-2 p_{B}^{2}}$, the recipient demands $t^{*}=D^{A}$. No MFP is formed, and the project is funded unless donor $A$ is politically constrained.

This proposition states that, first, the valuation ratio $\frac{D^{A}}{D^{B}}$ must be so high that the lower side payment is out of the question. Given this, if $A$ 's political constraints are stringent enough, then the large side payment that only an MFP would fund cannot be optimal. This is the case for sure if donor $B$ 's political constraints are stringent, so that $p_{B}>\frac{1}{2}$. Again, the intuition is that by choosing a large payment, the recipient would force the donors to form an MFP or forgo the project. If $p_{A}$ is very low relative to $p^{B}$, the incentive to rely on donor $A$ is more lucrative than the risky MFP strategy.

Consider finally the most interesting possibility, namely that an MFP is formed.

Proposition 3 (Large side payment). Suppose $\frac{D^{A}}{D^{B}}>\frac{\left(1-p_{B}\right) p_{A}+\left(1-p_{A}\right) p_{B}}{1-p_{A}}$ and $p_{A}<\frac{1-p_{B}\left(1-p_{B}\right)}{1-2 p_{B}^{2}}$. The following hold:

1. If $\frac{D^{A}}{D^{B}}>\frac{1-p_{B}}{p_{B}}$, the recipient demands $t^{*}=D^{A}$. No MFP is formed, and the project is funded unless donor $A$ is politically constrained.

2. If $\frac{D^{A}}{D^{B}}<\frac{1-p_{B}}{p_{B}}$, the recipient demands $t^{*}=D^{A}+D^{B}$. An MFP is formed, and the project is funded if and only if neither donor is politically constrained. 
In this case, we must consider two issues. First, an MFP cannot be formed if the safe offer, $t^{*}=D^{B}$, dominates the intermediate offer, $t^{*}=D^{A}$. This is not enough, however, as one must compare $D^{A}$ and $D^{A}+D^{B}$. If $p_{A}$ is sufficiently low so that the conditions for an automatic intermediate condition hold, then it is possible that the MFP is optimal. This is so when the probability that donor $B$ is constrained is also not prohibitively high, so that $\frac{D^{A}}{D^{B}}<\frac{1-p_{B}}{p_{B}}$. This condition can only hold if $p^{B}<\frac{1}{2}$. In this case, the value of securing MFP funding dominates the increased risk of project failure because the ratio $\frac{D^{A}}{D^{B}}$ obtains an intermediate value and the probability that donor $B$ is constrained is not very large. The condition is easier to meet for low values of $p_{B}$.

Figure 1 provides a graphical illustration of these conditions. The upper panel examines the case in which MFP formation is impossible due to a high probability of country $B$ being constrained. If $p_{B}>\frac{1}{2}$, the recipient never demands such a payment that only the MFP would fund. Given that $p_{B}>\frac{1}{2}$, we also have $\frac{1-p_{B}}{p_{B}}<1$. Therefore, the recipient's demand is either low $t^{*}=D^{B}$ or intermediate $t^{*}=D^{A}$, depending on whether the ratio $\frac{D^{A}}{D^{B}}$ is high enough to warrant some risk.

The middle panel presents a similar situation. Again, no MFP forms because of domestic constraints. However, now the problem is the high probability of the more enthusiastic country $A$ being constrained. Although $p_{B}<\frac{1}{2}$, the condition for choosing between the low and intermediate offers remains unchanged.

The lower panel finally examines the case in which MFP formation is possible. When the $\frac{D^{A}}{D^{B}}$ ratio obtains an intermediate value, MFP formation is ideal. For very high ratios $\frac{D^{A}}{D^{B}}$, the intermediate offer $t^{*}=D^{A}$ is optimal because country $A$ 's willingness to pay is high: why risk failure by insisting that country $B$ also participates when country $A$ is already paying a lot for the project? For very low ratios $\frac{D^{A}}{D^{B}}$, the low offer $t^{*}=D^{B}$ is optimal because it is safe. But for intermediate ratios $\frac{D^{A}}{D^{B}}$, the high offer $t^{*}=D^{A}+D^{B}$ due to a high reward-risk ratio.

[Figure 1 about here.]

The empirical implications of our model are testable. First, the recipient's demand for a given project should be higher when addressed to a multilateral institution than to an individual donor. Controlling for project size, the financial support that is requested by recipient countries should be higher for multilateral projects than compared to bilateral ones. This hypothesis can be tested by examining donors' share of project costs for bilateral and multilateral funding of public goods. 
Second, we expect that donors participating in MFPs attempt to tie their hands to reduce recipients' ability to extract rents. For example, donors could announce an explicit funding ceiling, so as to prevent recipients from blackmailing them. This hypothesis can be tested by examining episodes of MFP formation in light of the idea that donors have incentives to tie their own hands. From a comparative perspective, these incentives should be particularly pronounced for large multilateral organizations with many members and a mission to fund global public goods.

Finally, our model predicts that increased domestic political constraints in donor countries should result in increased relative importance of bilateral funding for global public goods. For example, financial hardship (Bulir and Hamann, 2008) or public opinion (Milner, 2006) could impose new domestic political constraints on funding for global public goods. While this should reduce funding for global public goods overall, we would expect this effect to be larger for multilateral than for bilateral funding. Data on the relationship between domestic political-economic developments in donor countries and their bilateral versus multilateral funding commitments would admit an empirical test.

The side payments enshrined in the Montreal Protocol on ozone depletion illustrate these results. In the initial text of the Montreal Protocol, the signatory countries agreed to reduce annual production and consumption of chlorofluorocarbons (CFCs) by 1998 by 50 per cent against a 1986 baseline. At the second Conference of the Parties (London, 1990) a Multilateral Fund for the Implementation of the Montreal Protocol was established to fund projects in developing countries that reduce ozone depletion. Since 1991, this fund has funded projects worth over US\$ 2,600 million. ${ }^{20}$ This case, we argue, illustrates MFP formation in response to recipient demands. Wealthy countries had a shared interest in combating ozone depletion, this required efforts in less enthusiastic developing countries, and political constraints were largely absent. Consider each claim in detail.

As Barrett (1999) notes, ozone depletion was to cause health problems from skin cancer to eye cataracts, as well as to reduce agricultural productivity, in the global North. Although industrialized countries' preferences initially diverged, "as a result of a growing perception of the vulnerability to ozone depletion in combination with advances in developing substitutes for CFCs, all [developed] states began gradually to perceive common interests in protecting the stratospheric ozone layer by phasing out harmful chemicals"

\footnotetext{
${ }^{20}$ See http: //www.multilateralfund.org/default.aspx. Accessed August 24, 2011.
} 
(Sprinz and Vaahtoranta, 1994, 95). In terms of our model, multiple potential donors ascribed a high value to combating ozone depletion. The developing countries, however, were concerned about the high cost of replacing CFCs with substitutes (Barrett, 1999). As DeSombre and Kauffman (1996, 94) put it, "[t]he developing country fear was that a worldwide shift to more expensive substitutes would increase the price and decrease the availability of CFCs." However, their efforts to prevent increased CFC use were important, especially in large countries such as China and India.

Given that industrialized countries had already agreed to reduce their emissions, and the Montreal Protocol was supported by major chemical companies both in the United States and Europe, political opposition to its implementation had largely disappeared (Benedick, 1998; Parson, 2003). In the 1990 London meeting, for example, the Bush administration had instructed the U.S. delegation to oppose the creation of a multilateral fund. But domestic NGOs, the chemical industry, other industrialized countries, and the developing countries resisted the move. Thus, "the Bush administration ultimately reversed itself and supported a fund" (DeSombre and Kauffman, 1996, 98). Domestic political constraints were no longer an excuse not to participate in funding, given the strong domestic support for the Montreal Protocol.

Importantly, our model sheds new light on the institutional design of funding arrangement for the Montreal Protocol. Barrett (2001) has previously argued that the Montreal Protocol is consistent with the notion that donors' side payments left each recipient indifferent between joining and not joining the Montreal Protocol. In other words, he interprets funding under the Montreal Protocol as pertaining to the "incremental" cost of emissions reductions. In reality, at the vigorous insistence of developing countries, the Multilateral Fund for the Implementation of the Montreal Protocol in Article 10 on the Financial Mechanism states that funding pertains to the "agreed incremental costs." ${ }^{21}$ While our model's prediction of full rent extraction by the recipients is also empirically inaccurate, it highlights the fact that the recipients were able to extract rents from the donors in the negotiations. ${ }^{22}$ This rent extraction, we have argued above, reflects the multilateral treaty's high collective valuation of global cooperation.

As to the possibility that MFP formation fails, the rather controversial nature of the multilateral ne-

\footnotetext{
${ }^{21}$ See http://ozone.unep.org/new_site/en/Treaties/treaty_text.php?treatyID=2\&secID=48. Own emphasis. Accessed October 2, 2011.

${ }^{22}$ While the concept of incremental costs denotes the difference in costs between the unilaterally and collectively optimal outcome, the Montreal Protocol's Multilateral Fund particularly emphasizes a focus on agreed incremental costs. This leaves room for bargaining over rents.
} 
gotiations on North-South finance for climate mitigation is instructive (Stewart, Kingsbury, and Rudyk, 2009). In spite of the importance of decarbonizing growth in rapidly industrializing developing countries, industrialized countries have just started to form an institutional architecture for climate mitigation. In the 2010 Cancun climate summit, a vague agreement on the aspirational goal of providing developing countries with US\$ 100 billion in climate finance was achieved. Despite more detailed agreement on the design of this Green Climate Fund at the Durban conference in 2011, the Bonn climate talks that ended on May 25, 2012 concluded without any "progress ... to deliver the financial support that the world's poorest and most valuable need to deal with the growing impacts of climate change." 23

Apart from this haggling about who is going to contribute what, the treaty text on the Green Climate Fund is not legally binding and strongly emphasizes adaptation and not mitigation. Given our argument, the focus on climate finance for adaptation is not surprising because benefits from adaptation are mostly local compared to global benefits from mitigation efforts. Mitigation is therefore prone to the strategic extraction of rents as demonstrated in our model, while less diffuse adaptation benefits make multilateral funding easier.

This failure to provide multilateral funding for climate mitigation is understandable in light of our model, given the large sums that effective climate mitigation finance would require and the fiscal constraints that industrialized countries struggling with weak economic performance and aging populations face. Additionally, donor countries' vulnerability to climate change varies greatly (Nordhaus, 2010), and our model suggests that this also presents difficulties for multilateral funding. The fact that bilateral finance initiatives have formed in spite of these domestic political constraints and different valuations of climate damages is also consistent with the model (Atteridge et al., 2009).

Interestingly, our model predicts that developing countries may moderate their demands in the future unless the domestic political constraints on climate finance are relaxed in potential donor countries. While this may not create a window of opportunity for meaningful multilateral climate finance, it may enable the formation of bilateral and minilateral finance arrangements, and thus contribute to climate mitigation in a bottom-up fashion.

\footnotetext{
${ }^{23}$ "Bonn Climate Talks End in Discord and Disappointment." Guardian May 25, 2012. See http: //www. guardian. co . uk/environment/2012/may/25/bonn-climate-talks-end-disappointment. Accessed May $28,2012$.
} 


\section{The Paradox of Multilateral Funding}

A possible counterargument to our modeling strategy pertains to the recipient's initiative. Given that MFP formation is a lengthy process that often results in a highly "sticky" organization (March and Olsen, 1998), it is of importance to examine how our conclusions change if the donors can form the MFP already before the recipient's demand. This model variant allows the donors to credibly commit to an institutional solution, and may thus increase their payoff.

Formally, consider the main model but suppose that each donor can, prior to the recipient's demand, credibly commit to not forming an MFP. The donors have yet to learn their own types, so suppose they maximize their ex ante payoff based on the prior probabilities $p_{A}, p_{B}$.

How does this credible commitment capacity influence equilibrium play? The solution to this modified game is simple: since the recipient extracts maximal surplus from an MFP in the main model, donors have strong incentives not to form an MFP.

Proposition 4 (Donor initiative and multilateral funding programs). If the donors can decide on forming an MFP prior to the recipient's offer, they do not form it.

Of the two donors, the less interested one, donor $B$, has particularly strong incentives to reject the MFP. By doing so, it can force the more interested donor $A$ to fund the global public good unilaterally. Thus, it obtains a free rider's payoff.

The result provides useful theoretical insights on donor incentives, yet we caution against a literal interpretation. Multilateral funding institutions already exist, so donors cannot choose to reject international institutions in a blanket fashion. To the extent that donors can constrain themselves by limiting multilateral funding, they benefit. This seems paradoxical given the collective action problems that pertain to global public goods. Bargaining interactions with recipients explain this paradox.

Notably, this extension is relevant for the difficulties of multilateral finance for climate mitigation. If donors have strong incentives to tie their own hands in regard to multilateral funding, so as to prevent the recipients' from upping the ante, it is not altogether surprising that even the most interested donors, such as the European Union, have not made a strong push for multilateral climate finance. Had they done so, our model extension predicts that they would have given possible recipients a considerable bargaining 
advantage. This would have increased the fiscal cost of climate mitigation finance.

In light of recent international climate negotiations by the Conference of the Parties in Cancun and Durban, our model gains tentative empirical support. One of the main issues in these negotiations has been how to set up a reliable climate finance infrastructure and how to acquire sufficient financial resources for it. ${ }^{24}$ Consistent with our model's expectations, donor countries such as the United States argue in favor of organizing climate finance mainly through private funds and in a bilateral fashion. Notwithstanding the need for "a combination of private and public sources, both bilateral and multilateral", the US proposal on longterm finance emphasizes the need to "substantially increase private investments in green infrastructure." 25

While the US administration may be an exception in its position on climate finance, Japan's Hatayama Initiative that was announced in September 2009 promises to "provide financial assistance to developing countries which are taking active emission reduction measures or those which are vulnerable to the negative impacts of climate change ..., including public and private financing." 26 This bilateral funding takes the form of grants, loans, and technical assistance, amounts to US\$15 billion, and is to about a quarter financed by private sources. ${ }^{27}$ On a similar note, in 1994, the French government decided to split off from the 1991 created Global Environment Facility $(\mathrm{GEF})^{28}$, the largest environmental funding organization to date. Instead of providing environmental funding for projects in climate change, biodiversity, or land degradation exclusively through the multilateral channels of the GEF, the French government established the French Global Environmental Facility (FGEF) as its own funding instrument to provide bilateral environmental funding. ${ }^{29}$ Since creating and running the FGEF that disbursed bilateral environmental assistance of Euro 354 million since its initiation is costly, our model may provide a rationale on why to operate your own funding organization. Apart from better control of targeting funds for global public good provision, we would argue that bilateral finance instruments guard donor countries against strategically increased transfer payment requests

\footnotetext{
${ }^{24}$ See http: / / www. economist.com/blogs/newsbook/2011/12/climate-change-0. Accessed December 20, 2011.

${ }^{25}$ See http: //unfccc.int/resource/docs/2011/awglca14/eng/crp35.pdf. Own emphasis. Accessed December 20, 2011.

${ }^{26}$ See http: //www.mofa.go.jp/policy/oda/white/2009/html/honbun/b1/s3_1.html. Accessed May 30, 2012.

${ }^{27}$ Further information on this funding program, application procedures, eligibility criteria, and project types can be found at http://www.climatefinanceoptions.org/cfo/node/63.

${ }^{28}$ See http: / / www . thegef . org/gef/home for further information. Accessed May 30, 2012.

${ }^{29}$ For additional information on the FGEF, see http://www.ffem.fr/lang/en/accueil/ffem. Accessed May 30, 2012.
} 
by recipient countries. The case of the French Global Environmental Facility may subscribe to this logic.

This pronounced focus of donor countries on bilateral funding contrasts with the Indian submission to the Ad-Hoc Working Group on Long-term Cooperative Action under the Convention, which emphasizes "the coordination of all current and future funds" and seeks to "advance and enable such coordination in order to improve the effectiveness of these funds through rationalization measures." 30 Donor countries strive for bilateral finance infrastructure that builds on private investments, while recipient countries favor multilateral mechanisms with coordinated action.

This illustrates our model's key finding: multilateral financing of global public goods primarily benefits recipient countries. All else constant, a recipient country extracts more rents under a multilateral scheme than under a bilateral scheme. This practice is inefficient because if recipients spend a lot of money in any particular project, they have fewer resources to implement other projects. Overall, global environmental benefits from project funding decrease..$^{31}$

\section{The Strategic Use of Funding Constraints}

If donors have incentives to tie their hands, one may surmise that they would also benefit from funding constraints on an MFP. Suppose, indeed, that if the donors do create an MFP in the main model, they are able to impose a ceiling, denoted by $T>0$, on multilateral funding. ${ }^{32}$ This ceiling basically states that demands beyond it are automatically rejected by the MFP staff. How would such a funding constraint influence equilibrium behavior?

Proposition 5 (Funding constraints). Suppose the MFP has a funding constraint, T. The following hold:

1. If the recipient did not demand $t^{*}=D^{A}+D^{B}$ in the main model, equilibrium behavior is unchanged.

2. If the recipient demanded $t^{*}=D^{A}+D^{B}$ in the main model and the funding constraint is sufficient lax, the recipient demands $T$ instead.

\footnotetext{
${ }^{30}$ See http: //unfccc.int/resource/docs/2011/awglca14/eng/crp04.pdf. Accessed December 20, 2011.

${ }^{31}$ We do not imply that the side payments to recipient countries be themselves non-legitimate. Rather, we highlight the strategic incentives that recipients have to request higher side payments from MFPs than from bilateral programs.

${ }^{32} \mathrm{We}$ assume that introducing a ceiling for project funding does not reveal information about the donors' Bayesian types to the recipient country. Hence, the recipient cannot update her beliefs by observing the donors' self-imposed funding commitments in the MFP. This is a reasonable assumption because most MFPs are implemented within the confines of extant international organizations with institutional rules that are difficult to modify for any given project. Thus, the funding constraints can be treated as exogenous for any given project funding decision.
} 
3. If the recipient demanded $t^{*}=D^{A}+D^{B}$ in the main model and the funding constraint is sufficiently stringent, the recipient demands $D^{A}$ instead.

If the donors can limit the funding available to the MFP before the game begins, their expected payoff increases whenever the recipient demanded $D^{A}+D^{B}$ in the main model.

Clearly, if the recipient previously had no incentive to demand multilateral funding, nothing changes. From the recipient's perspective, the MFP is now even less lucrative than previously. But if the recipient previously relied on the MFP, then the size of the funding constraint is key. As long as the funding constraint is relatively lax, so that $T$ is not much lower than $D^{A}+D^{B}$, the recipient continues to demand multilateral funding. Yet the donors now obtain some of the benefits. If $T$ is too stringent, the value of multilateral funding no longer outweighs the risk associated with one donor rejecting the MFP. Thus, the recipient demands $D^{A}$ instead.

The empirical importance of such funding constraints is illustrated by the heated arguments concerning the "additionality principle" in multilateral environmental funding, such as that provided by the Global Environmental Facility (Clémençon, 2006). Ever since the 1992 Earth Summit, wealthy donors have insisted that environmental foreign aid be based on the incremental costs of the project, so that the recipients receive none of the rents from project funding. Our model extension shows that imposing such a constraint on an MFP, such as the Global Environmental Facility, may result in recipients' demanding funding through bilateral channels.

The strategic use of funding constraints seems relevant to the Climate Investment Funds of the World Bank. ${ }^{33}$ Formed in May 2008, the Clean Technology Fund and the Strategic Climate Fund promote clean technology and decarbonization in developing countries. Already in September 2008, donor countries announced a total contribution of approximately US\$ 6.1 billion for these funds. ${ }^{34}$. The announcement of a fixed quantity of funding, without decisions on specific projects, is consistent with donors' incentives to restrain themselves.

This extension also shows that even if the donors were able to dictate the terms of multilateral project funding ex ante, participation would present a problem. Suppose, indeed, that the donors could form an

\footnotetext{
${ }^{33}$ See http: / / www. climateinvestmentfunds.org/cif/. Accessed August 22, 2011.

${ }^{34}$ See http: / / go. worldbank. org/36H73DPMV0. Accessed August 22, 2011
} 
MFP before the recipient moves and that the MFP could induce the recipient to implement a project in exchange for the minimal incentive-compatible side payment, $t=C$. Suppose also donor $B$ believed that donor $A$ is probably not constrained, so that $p_{A}$ is very low. In this case, donor $B$ is almost certain to obtain a zero payoff if it rejects the MFP because the recipient would propose the project for donor $A$ to fund. By

forming the MFP, donor $B$ would have to pay $\frac{1}{2}$ of the minimal amount of funding that induces the recipient to implement, or $t=C$. Thus, even if the donors dominated bargaining, they would not form an MFP. This reasoning shows that our main result is not driven by the assumption that the recipient holds the initiative.

\section{Donor Private Information}

In the main model, there was no uncertainty between the donors. If we relax this assumption, strategic behavior is complicated because if the recipient demands $t^{*}=D^{B}$, each donor may choose to "gamble" by not funding in the hope that the other will fund it. A full analysis of this issue results in complexity because one needs to apply mechanism design techniques to obtain a full characterization. However, the following general result holds:

Proposition 6 (Donor private information). If a donor's type is her private information, then the recipient's expected payoff from demanding $t^{*}=D^{A}$ or $t^{*}=D^{A}+D^{B}$ remains unchanged. However, the recipient's payoff from demanding $t^{*} \leq D^{B}$ decreases relative to the main model.

Uncertainty among donors increases the recipient's incentive to demand large concessions. This reduces the probability of project implementation and results in a lower payoff to all players.

\section{Conclusion}

Many global public goods are provided through national implementation of projects and policies. Unless national governments are completely altruistic, they fail to provide many global public goods with an excellent global cost-benefit ratio because domestic costs exceed domestic benefits.

We have examined this problem with a particular focus on three factors: collective action, domestic political constraints in donor countries, and recipients' efforts to extract maximal concessions from donors. Our 
primary finding is that international organizations funding global public goods enhance recipients' ability to extract concessions from donors, and this implies that donors have incentives to reject such institutions. ${ }^{35}$ In the case of global public goods, multilateralism is an income transfer from donors to recipients.

We also find that donors with severe domestic political constraints on funding can free ride on other donors' contributions. This is so even if multilateral funding is enforceable under the auspices of a multilateral funding program. Such domestic political constraints reduce a recipient's payoff from interactions with donors, and funding constraints in multilateral funding programs have similar effects.

These findings have important policy implications. North-South provision for global public goods has proven a difficult political question, not least in the discussion of an institutional architecture for climate mitigation finance. Our model indicates that donors have incentives to refrain from creating multilateral funding institutions. Such institutions internalize the global benefits of global public goods, and so they are not in a position to reject tough demands for funding by the recipients. Donor countries' opposition to institutions can be mitigated through the imposition of funding constraints.

To be sure, our model downplays some other important issues, such as the effect of "problem structure" on cooperation (Mitchell and Keilbach, 2001). For simplicity, we have not tried to examine how our model's conclusions depend on the exact problem structure. However, it seems plausible that our model applies best to problems characterized by deep North-South asymmetry, so that the identities of donors and recipients are, at least for any given project, clear.

On a deeper theoretical level, the model highlights a frequently neglected dimension of international politics: countries' incentives to constrain their ability to engage in collective action. One might imagine, as many prominent cooperation theorists have done (Abbott and Snidal, 1998; Barrett, 2003; Keohane, 1984), that countries generally have a clear interest in collective action but achieving it is difficult. This may well be so on the highest levels of abstraction, but in practice some groups of countries may want to restrain their own ability to cooperate, so as to influence the behavior of outsiders. In our model, donors have incentives to reduce their ability to create international institutions that ultimately empower recipients.

\footnotetext{
${ }^{35}$ As an alternative to our conclusion that donor countries reject multilateral funding institutions, they may exert other forms of control to protect themselves against rent extraction by recipient countries. One-dollar, one-vote decision-making rules in the International Bank for Reconstruction and Development are but one example.
} 


\section{Mathematical Appendix: Equilibrium Characterization}

\section{Nash Bargaining Solution}

As described in the text above if $M_{A}=M_{B}=1$, i.e., if the MFP is formed, we assume the donor countries $A$ and $B$ to play a Nash (1950) Bargaining Game. In a Nash Bargaining Game, each donor $i$ simultaneously proposes a share $s_{i} \in[0,1]$. If $s_{A}+s_{B} \leq 1$, then bargaining succeeds. Otherwise it fails and "disagreement" occurs. Without cooperation, the damages the two donor countries incur are $-D^{A}$ and $-D^{B}$, which are thus the disagreement points. With cooperation, the donors must agree on burden sharing to fund $t^{*}$ but damages are avoided. Clearly, any allocation such that $s_{A}+s_{B}=1$ is a Nash equilibrium.

To find the Nash Bargaining Solution requires the maximization of the Nash product $\left(U^{A}-U_{0}^{A}\right)\left(U^{B}-\right.$ $\left.U_{0}^{B}\right)$, where $U^{A}$ and $U^{B}$ are utility levels from cooperation, while $U_{0}^{A}$ and $U_{0}^{B}$ are utility levels at the disagreement points. Plugging disagreement points into the Nash product equation and noting that $U^{B}=$ $-t-U^{A}$ needs to hold because $s_{A}+s_{B}=1$ given that Nash bargaining is conceptualised as a zero-sum game on the Pareto frontier, we face the following maximization problem:

$$
\max _{U^{A}}\left(U^{A}-\left(-D^{A}\right)\right)\left(-t-U^{B}-\left(-D^{B}\right)\right)
$$

Multiplying out and taking the first-order derivative with respect to $U^{A}$ yields

$$
-t+D^{B}-D^{A}=2 U^{A}
$$

as first-order condition. Clearly, this can be re-written as $U^{A}=-D^{A}+\frac{1}{2}\left(-t+D^{A}+D^{B}\right)$, which is identical to what we have in equation (1). Due to symmetry of the game, we get $U^{B}=-D^{B}+\frac{1}{2}\left(-t+D^{B}+D^{A}\right)$ for donor $B$. The payoff from cooperation for each donor is thus the difference between half of the added value of funding the project and the disagreement point.

\section{Complete Information Model}

With complete information, we apply subgame-perfect Nash equilibrium as solution concept and solve the game by backward induction. Hence, we first consider what has been called "MFP formation subgame" in 
the main text. In this game the two donors $A$ and $B$ simultaneously decide if to participate in the MFP, if to fund the project unilaterally, or not to implement the project at all. Since a Nash equilibrium results from mutual best responses we have the following for donor country $A$.

Assume that $B$ participates in the MFP, i.e., $M_{B}=1$. Then $A$ has an incentive to participate as well if her payoff from MFP formation is at least as high as payoffs from unilateral provision (equation 6a) or no project implementation at all (equation $6 b$ ). This requires

$$
\begin{aligned}
-\frac{1}{2} D^{A}+\frac{1}{2} D^{B}-\frac{1}{2} t^{*} \geq-t^{*} & \Leftrightarrow \quad D^{A}-D^{B} \leq t^{*} \\
-\frac{1}{2} D^{A}+\frac{1}{2} D^{B}-\frac{1}{2} t^{*} \geq-D^{A} & \Leftrightarrow \quad t^{*} \leq D^{A}+D^{B} .
\end{aligned}
$$

If we instead consider $B$ 's non-participation with $M_{B}=0$, then $A$ does not have to take a participation decision because no MFP can form. However, $A$ can fund the project unilaterally, which is obviously rational if the side payment to the recipient is (weakly) smaller than $A$ 's damage from non-implementation. Unilateral funding, i.e., $F_{A}=1$ maximizes $A$ 's utility if $t^{*} \leq D^{A}$, absent an MFP.

$A$ 's best-response for MFP formation can, therefore, be written as

$$
M_{A}= \begin{cases}1 & \text { if } D^{A}-D^{B} \leq t^{*} \leq D^{A}+D^{B} \\ 0 & \text { otherwise }\end{cases}
$$

Now, we derive the best-response function for $B$. Again, assume first that $A$ participates in the MFP $\left(M_{A}=1\right)$ and is willing to fund unilaterally if $t^{*} \leq D^{A}$. While we allow the more ambitious donor to fund the project unilaterally, we exclude this unilateral funding alternative for the less ambitious donor $D^{B}$. This assumption is, however, an innocuous one as under complete information, the recipient will never implement the project with the less ambitious donor due to $D^{A}>D^{B}$. Because of higher valuations for the project by $A$, the recipient always targets donor $A$, in equilibrium, because she can extract more rents from $A$ than from $B$. A potentially optimal side payment $t^{*}=D^{B}$ is always strictly dominated by $t^{*}=D^{A}$ and, therefore, our equilibrium results are not affected by this assumption.

Given this, B's participation in an MFP is optimal if the payoffs from MFP formation outweigh the payoffs from free-riding (equation 8) and from non-implementation (equation 9). Free-riding is possible if 
the recipient's optimal offer $t^{*}$ is such that the project could be implemented unilaterally by donor $A$. Thus, if $t^{*} \leq D^{A}$, participation is optimal iff

$$
-\frac{1}{2} D^{B}+\frac{1}{2} D^{A}-\frac{1}{2} t^{*} \geq 0 \quad \Leftrightarrow \quad t^{*} \leq D^{A}-D^{B}
$$

If $t^{*}>D^{A}$, so that the project cannot be funded unilaterally and would fail without MFP formation, participation is utility maximizing for $B$ iff

$$
-\frac{1}{2} D^{B}+\frac{1}{2} D^{A}-\frac{1}{2} t^{*} \geq-D^{B} \quad \Leftrightarrow \quad t^{*} \leq D^{A}+D^{B}
$$

In case of $A$ 's non-participation, $B$ does not need to take a participation decision as an MFP cannot be formed anyways. Also, $B$ cannot fund unilaterally, which gives the following best-response for the less ambitious donor $B$.

$$
M_{B}= \begin{cases}1 & \text { if } t^{*} \leq D^{A}-D^{B} \text { or } D^{A}<t^{*} \leq D^{A}+D^{B} \\ 0 & \text { otherwise. }\end{cases}
$$

From these mutual best-responses, it follows immediately that we find two Nash equilibria in the MFP formation subgame. One equilibrium occurs for optimal recipient offers $t^{*}$ that fall into the interval $\left(D^{A} ; D^{A}+D^{B}\right]$, while another equilibrium exists for a side payment $t^{*}=D^{A}-D^{B}$. Note, however, that the second equilibrium is a result of the implicit assumption that weak inequalities are sufficient to constitute MFP formation. This equilibrium, is not robust to minor perturbations in payoffs and thus not trembling-hand perfect. Again, this is more of technical than of substantive interest as this equilibrium to the MFP formation subgame is not an equilibrium for the complete game.

As the recipient seeks to maximize utility, which is the difference between the side payment $t^{*}$ and the cost of project implementation $C$, the recipient demands an optimal side payment $t^{*}=D^{A}+D^{B}$. The complete information subgame-perfect Nash equilibrium is characterized by $\left\{t^{*}=D^{A}+D^{B} ;\left(M_{A}=\right.\right.$ $\left.\left.1, M_{B}=1\right)\right\}$.

In equilibrium, the recipient forces the donors $A$ and $B$ to form an MFP and extracts the highest possible rent. The payoff for the recipient is $D^{A}+D^{B}-C$, whereas $A$ yields $-D^{A}$ and $B$ yields $-D^{B}$. In equilibrium, the donors are indifferent between MFP formation and the non-implementation of the project. 


\section{Incomplete Information Model}

With incomplete information, we resort to the perfect Bayesian Nash equilibrium as solution concept for our game. Again, we first have a look at the MFP formation subgame. Despite the introduction of uncertainty about domestic political constraints, both donor countries' participation decisions remain unchanged. This strategic rationale holds as we assume that player types are revealed prior to the donors' participation decision. Hence, incomplete information exclusively pertains to the recipient country. The recipient country maximizes expected utilities and chooses an equilibrium strategy that is consistent with her beliefs $p_{A}$ and $p_{B}$ as the probabilities of domestic opposition. For the recipient to rationally request an aggressive demand $t^{*}=D^{A}+D^{B}$ instead of $t^{*}=D^{A}$ it must hold that

$$
\begin{gathered}
D^{A}\left(1-p_{A}\right)\left(1-p_{B}\right)+D^{A}\left(1-p_{A}\right) p_{B} \leq\left(D^{A}+D^{B}\right)\left(1-p_{A}\right)\left(1-p_{B}\right) \\
\Leftrightarrow \quad \frac{D^{A}}{D^{B}} \leq \frac{1-p_{B}}{p_{B}} \quad \text { if } p_{B} \neq 0 .
\end{gathered}
$$

For the aggressive offer $t^{*}=D^{A}+D^{B}$ to be rational compared to the lowest side payment request $t^{*}=D^{B}$, we need to have

$$
\begin{array}{r}
D^{B}\left(1-p_{A}\right)\left(1-p_{B}\right)+D^{B}\left(1-p_{B}\right) p_{A}+D^{B}\left(1-p_{A}\right) p_{B} \leq\left(D^{A}+D^{B}\right)\left(1-p_{A}\right)\left(1-p_{B}\right) \\
\Leftrightarrow \quad \frac{\left(1-p_{B}\right) p_{A}+\left(1-p_{A}\right) p_{B}}{\left(1-p_{A}\right)\left(1-p_{B}\right)} \leq \frac{D^{A}}{D^{B}} \quad \text { if } p_{A} \neq 1 \text { and } p_{B} \neq 1 .
\end{array}
$$

And finally if

$$
\begin{gathered}
D^{B}\left(1-p_{A}\right)\left(1-p_{B}\right)+D^{B}\left(1-p_{B}\right) p_{A}+D^{B}\left(1-p_{A}\right) p_{B} \leq D^{A}\left(1-p_{A}\right)\left(1-p_{B}\right)+D^{A}\left(1-p_{A}\right) p_{B} \\
\Leftrightarrow \quad \frac{\left(1-p_{B}\right) p_{A}+\left(1-p_{A}\right) p_{B}}{1-p_{A}} \leq \frac{D^{A}}{D^{B}} \quad \text { if } p_{A} \neq 1
\end{gathered}
$$

holds, then the recipient chooses $t^{*}=D^{A}$ over $t^{*}=D^{B}$. These three conditions fully describe the recipient's equilibrium strategy as a function of the recipient's beliefs about $p_{A}$ and $p_{B}$ as well as the damage ratio $\frac{D^{A}}{D^{B}}$. This strategy together with the donors' participation strategies in the MFP formation subgame constitute the perfect Bayesian Nash equilibria. 


\section{Mathematical Appendix: Proofs for the Main Model}

Proof of Proposition 1. Suppose $\frac{D^{A}}{D^{B}}<\frac{\left(1-p_{B}\right) p_{A}+\left(1-p_{A}\right) p_{B}}{1-p_{A}}$. In this case, condition 13 cannot hold, so $t=D^{B}$ dominates $t=D^{A}$. If this condition holds, the condition 12 also cannot hold because $1-p_{B}$ in the denominator obtains a value less than unity. Thus, $t^{*}=D^{B}$ is optimal. Given that the project is funded even when donor $B$ fails to participate in the MFP, no MFP is formed. Donor $A$ funds the project by the equilibrium refinement.

Proof of Proposition 2. Suppose $\frac{D^{A}}{D^{B}}>\frac{\left(1-p_{B}\right) p_{A}+\left(1-p_{A}\right) p_{B}}{1-p_{A}}$. If $p_{B}>\frac{1}{2}$, then condition 11 cannot hold, and thus $t^{*}=D^{A}$. As shown in the equilibrium analysis, no MFP is formed but the project is funded whenever donor $A$ is not politically constrained. If $p_{A}>\frac{1-p_{B}\left(1-p_{B}\right)}{1-2 p_{B}^{2}}$, then condition 12 cannot be met because $\frac{1-p_{B}}{p_{B}}<\frac{\left(1-p_{B}\right) p_{A}+\left(1-p_{A}\right) p_{B}}{\left(1-p_{A}\right)\left(1-p_{B}\right)}$ and thus the condition for $t=D^{A}+D^{B}$ dominating $t=D^{A}$ cannot be met for any $\frac{D^{A}}{D^{B}}$ such that $t=D^{B}$ would not be optimal.

Proof of Proposition 3. Suppose $\frac{D^{A}}{D^{B}}>\frac{\left(1-p_{B}\right) p_{A}+\left(1-p_{A}\right) p_{B}}{1-p_{A}}$. Then $t=D^{A}$ must dominate $D^{B}$. Thus, it suffices to consider the expected payoff from $t=D^{A}+D^{B}$ and $t=D^{A}$. Suppose now $p_{A}<\frac{1-p_{B}\left(1-p_{B}\right)}{1-2 p_{B}^{2}}$. Now condition 11 holds for $\frac{D^{A}}{D^{B}}$ between $\frac{\left(1-p_{B}\right) p_{A}+\left(1-p_{A}\right) p_{B}}{1-p_{A}}$ and $\frac{1-p_{B}}{p_{B}}$, but fails otherwise. Thus, the recipient's optimal demand is as characterized in the proposition. The conditions for formation of an MFP and project funding follow directly from the equilibrium analysis.

\section{Mathematical Appendix: Robustness}

\section{Endogenous Disagreement Points}

For simplicity, in the main text we assumed the disagreements points from Nash Bargaining are $-D^{A}$ and $-D^{B}$. Does our equilibrium exist if we instead allow each donor $i$ to offer unilateral funding upon bargaining failure? To investigate this question, it is clear that we only need to focus on the MFP formation subgame. After all, the question is trivial unless an MFP is formed.

Suppose now an MFP has been formed. Assume the disagreement points reflect an additional subgame wherein $A$ and $B$ simultaneously accept or reject the recipient's prior offer $t$. Recall that in our equilibrium, an MFP is formed only if $t>D^{A}$. Given this, it is clear that neither $A$ nor $B$ would offer unilateral 
funding given the prior demand $t$. Thus, an equilibrium of the MFP subgame exists that produces identical equilibrium behavior and payoffs as in the main model. Similarly, it is clear that in the subgame without MFP formation equilibrium behavior and payoffs are identical. Therefore, the recipient's optimal offer $t^{*}$

must be unchanged. This means that our equilibrium exists even with endogenous disagreements points, so that our simplification innocuous.

\section{Domestic Constraints Unknown to Donors}

In the main text, we introduced uncertainty about domestic constraints under the assumption that this uncertainty only pertains to recipients; donor countries knew about their types, as domestic political constraints were assumed to be revealed prior to the donors' participation decision. In this section, we show that the same equilibria are sustained when we relax this assumption. We have shown that in the incomplete information game above, a recipient country chooses between an aggressive, a medium, and a low demand $t^{*}$ depending on its prior beliefs, $p_{A}$ and $p_{B}$, about the donor countries' domestic political constraints.

When we drop the assumption that donor countries know about the domestic constraints with certainty, the basic logic of the equilibrium characterization remains the same. Let there be governmental prior beliefs $p_{A} \in[0,1]$ and $p_{B} \in[0,1]$, which denote the probabilities in donor countries $A$ and $B$ that MFP formation or unilateral funding fail due to domestic opposition.

As before, let us first assume that donor $B$ participates in the MFP, i.e., that $M_{B}=1$ holds. With uncertainty about domestic opposition, $p_{A}, A$ then participates if her payoff from MFP formation is at least as high as payoffs from unilateral provision or no project implementation at all. Adjusting equations (6a) and $(6 \mathrm{~b})$ for uncertainty and noting that $p_{A} \neq 1$, gives

$$
\begin{gathered}
\left(1-p_{A}\right)\left(-\frac{1}{2} D^{A}+\frac{1}{2} D^{B}-\frac{1}{2} t^{*}\right)+p_{A} \cdot 0 \geq\left(1-p_{A}\right)\left(-t^{*}\right)+p_{A} \cdot 0 \quad \Leftrightarrow \quad D^{A}-D^{B} \leq t^{*} \\
\left(1-p_{A}\right)\left(-\frac{1}{2} D^{A}+\frac{1}{2} D^{B}-\frac{1}{2} t^{*}\right)+p_{A}\left(-D^{A}\right) \geq-D^{A} \quad \Leftrightarrow \quad t^{*} \leq D^{A}+D^{B} .
\end{gathered}
$$

This illustrates that despite the introduction of uncertainty about domestic constraints for the donor country $A$, the derived participation conditions remain the same. Hence, country $A$ 's best-response for MFP participation is identical to the one derived under the assumption that donor $A$ 's player type is revealed prior 
to the participation decision. The best-response is again given as presented in equation (7).

Drawing again on the assumption that only the more ambitious donor $A$ is allowed to fund the project

unilaterally, country $B$ only participates in MFP formation if the payoffs from multilateral funding outweigh the payoffs from free-riding and from non-implementation. Adjusting equations (8) and (9) for donors' incomplete information and noting that $p_{B} \neq 1$ yields

$$
\left(1-p_{B}\right)\left(-\frac{1}{2} D^{B}+\frac{1}{2} D^{A}-\frac{1}{2} t^{*}\right)+p_{B} \cdot 0 \geq 0 \quad \Leftrightarrow \quad t^{*} \leq D^{A}-D^{B}
$$

and

$$
\left(1-p_{B}\right)\left(-\frac{1}{2} D^{B}+\frac{1}{2} D^{A}-\frac{1}{2} t^{*}\right)+p_{B}\left(-D^{B}\right) \geq-D^{B} \quad \Leftrightarrow \quad t^{*} \leq D^{A}+D^{B} .
$$

This again leaves country $B$ 's best-response unchanged as compared to the expression which we presented in equation (10) above. These derivations show that even when introducing the possibility that donor countries are uncertain about domestic constraints with some positive probabilities $p_{A}, p_{B} \in[0,1)$, equilibrium play remains unaffected. As donor countries do not adjust their behavior in the MFP formation subgame, the optimal strategies of the recipient country does not change either.

\section{Mathematical Appendix: Model Extensions}

We present here three extensions of our main model.

\section{The Paradox Multilateral Funding}

It suffices to show that at least one donor has an incentive to credibly commit to not forming an MFP. Thus, simply consider donor $B$ to prove Proposition 4 :

Proof of Proposition 4. There are two possibilities to consider:

1. If the parameters of the game are such that the recipient's optimal offer in the main model is $t^{*}=D^{A}$ or $t^{*}=D^{B}$, then the initial commitment decision is irrelevant because the recipient would not demand $t=D^{A}+D^{B}$ in any case. 
2. If the parameters of the game are such that the recipient's optimal offer in the main model is $t^{*}=$ $D^{A}+D^{B}$, then each donor's expected payoff is $-D^{i}$ in the main model. A credible commitment to not forming an MFP can only result in a weakly higher payoff.

Thus, the proposition is proven.

\section{The Strategic Use of Funding Constraints}

Suppose now that the recipient cannot demand a larger side payment than $T$, where $T \in\left(D^{A}, D^{A}+D^{B}\right)$. Proposition 5 can be proven as follows:

Proof of Proposition 5. If the recipient's optimal demand was $t^{*}=D^{A}$ or $t^{*}=D^{B}$ in the main model, then reducing the MFP demand from $D^{A}+D^{B}$ to $T$ has no effect on the recipient's optimal demand. But if the recipient's (strictly) optimal demand was $t^{*}=D^{A}+D^{B}$, then there exists some threshold $\tilde{T}$ such that for all $T>\tilde{T}$ the optimal demand remains $t^{*}=D^{A}+D^{B}$ and for all $T<\tilde{T}$ the optimal demand changes to $t^{*}=D^{A}$ as $t=D^{B}$ cannot dominate $t=D^{A}$ if $t^{*}=D^{A}+D^{B}$ was optimal in the main model. Now note that each donor's expected payoff in the main model is $-D^{i}$ whenever $t^{*}=D^{A}+D^{B}$ is optimal. Their expected payoff is also a strictly decreasing function of $T$ because the probability of successful project implementation remains unchanged while the value to be distributed is a strictly decreasing function of $T$. Thus, the proposition holds.

\section{Donor Private Information}

Suppose now that each donor's type is her private information. Given this, we can prove Proposition 6 as follows:

Proof of Proposition 6. In this case, one should first note that if the recipient's offer is $t^{*}=D^{A}$ or $t^{*}=$ $D^{A}+D^{B}$, equilibrium behavior in the relevant subgame remains unchanged. An unconstrained donor $B$ rejects the MFP if the offer is $t^{*}=D^{A}$ given that $D^{B}<D^{A}$. An unconstrained donor $B$ accepts the MFP by our equilibrium selection rule. A constrained donor $B$ rejects the MFP. Similarly, donor $A$ 's behavior remains unchanged. The recipient also cannot demand in any equilibrium $t>D^{B}$ except for $t=D^{A}$ or $t=D^{A}+D^{B}$, because any such offer would be strictly dominated by $t^{*}=D^{A}$ or $t^{*}=D^{A}+D^{B}$ : 
this is shown in the equilibrium analysis above and the equilibrium analysis continues to hold given that equilibrium behavior is unchanged.

Suppose now that the recipient offers some $t \leq D^{B}$. Given incomplete information, each donor's strategic behavior is potentially complicated. It is plausible to assume that the donors can engage in costless "cheap talk" so that each selects a message $m \in[0, \infty)$. This costless communication allows the construction of a direct mechanism. As Myerson (1979) shows, such a direct mechanism can be used to produce the expected payoffs and actions that any indirect mechanism would produce. With this analytical apparatus in place, it remains to prove the proposition. To do this, note that the recipient's expected payoff cannot exceed $D^{B}-C$ in any circumstance. This is the expected payoff from offering $D^{B}$ in the main model, so the recipient's expected payoff must decrease. Thus, the set of parameters such that $D^{B}$ is optimal shrinks while the choice between $D^{A}$ and $D^{A}+D^{B}$ remains unchanged. This concludes the proof of the proposition. 


\section{References}

Abbott, Kenneth W., and Duncan Snidal. 1998. "Why States Act through Formal International Organizations." Journal of Conflict Resolution 42 (1): 3-32.

Agrawala, Shardul, and Steinar Andresen. 1999. "Indispensability and Indefensibility? The United States in the Climate Treaty Negotiations." Global Governance 5 (4): 457-482.

Anand, P.B. 2004. "Financing the Provision of Global Public Goods.” World Economy 27 (2): 215-237.

Atteridge, Aaron, Clarisse Kehler Siebert, Richard J. T. Klein, Carmen Butler, and Patricia Tella. 2009. "Bilateral Finance Institutions and Climate Change: A Mapping of Climate Portfolios." Stockholm Environment Institute.

Barrett, Scott. 1994. "Self-Enforcing International Environmental Agreements." Oxford Economic Papers 46 (Supplement): 878-894.

Barrett, Scott. 1999. "Montreal versus Kyoto: International Cooperation and the Global Environment.” In Global Public Goods: International Cooperation in the 21st Century, ed. Inge Kaul, Isabelle Grunberg, and Marc Stern. New York: Oxford University Press.

Barrett, Scott. 2001. “International Cooperation for Sale.” European Economic Review 45 (10): 1835-1850.

Barrett, Scott. 2003. Environment and Statecraft: The Strategy of Environmental Treaty-Making. Oxford: Oxford University Press.

Barrett, Scott. 2007. Why Cooperate? The Incentive to Supply Global Public Goods. Oxford: Oxford University Press.

Benedick, Richard E. 1998. Ozone Diplomacy: New Directions in Safeguarding the Planet. Cambridge: Harvard University Press.

Bueno de Mesquita, Bruce, and Alastair Smith. 2007. "Foreign Aid and Policy Concessions." Journal of Conflict Resolution 51 (2): 251-284.

Bulir, Ales, and A. Javier Hamann. 2008. "Volatility of Development Aid: From the Frying Pan into the Fire?" World Development 36 (10): 2048-2066.

Carraro, Carlo, and Domenico Siniscalco. 1993. "Strategies for the International Protection of the Environment." Journal of Public Economics 52 (3): 309-328.

Chong, Alberto, and Mark Gradstein. 2008. "What Determines Foreign Aid? The Donors' Perspective." Journal of Development Economics 87 (1): 1-13.

Clémençon, Raymond. 2006. "What Future for the Global Environment Facility?" Journal of Environment and Development 15 (1): 50-74.

DeSombre, Elizabeth R., and Joanne Kauffman. 1996. "The Montreal Protocol Multilateral Fund: A Partial Success Story." In Institutions for Environmental Aid: Pitfalls and Promise, ed. Robert O. Keohane, and Marc A. Levy. Cambridge: MIT Press. 
Drazen, Allan. 2002. "Conditionality and Ownership in IMF Lending: A Political Economy Approach." IMF Staff Papers 49: 36-67.

Fischer, Carolyn, and Richard G. Newell. 2008. "Environmental and Technology Policies for Climate Mitigation.” Journal of Environmental Economics and Management 55 (2): 142-162.

Goeschl, Timo, and Timothy Swanson. 2002. "The Social Value of Biodiversity R\&D.” Environmental and Resource Economics 22 (4): 477-504.

Hagen, Rune Jansen. 2006. "Samaritan Agents? On the Strategic Delegation of Aid Policy." Journal of Development Economics 79 (1): Hagen2006.

Harstad, Bård. 2011. "Buy Coal? Deposit Markets Prevent Carbon Leakage.” International Environmental Policy Making and Agreements. April 8-9, 2011 at Yale University.

Hicks, Robert L., Bradley C. Parks, J. Timmons Roberts, and Michael J. Tierney. 2008. Greening Aid? Understanding the Environmental Impact of Development Assistance. New York: Oxford University Press.

Hoel, Michael, and Kerstin Schneider. 1997. "Incentives to Participate in an International Environmental Agreement." Environmental and Resource Economics 9 (2): 153-170.

Iida, Keisuke. 1993. "When and How Do Domestic Constraints Matter? Two-Level Games with Uncertainty." Journal of Conflict Resolution 37 (3): 403-426.

Kapur, Devesh. 2002. "The Common Pool Dilemma of Global Public Goods: Lessons from the World Bank's Net Income and Reserves.” World Development 30 (3): 337-354.

Kaul, Inge, Isabelle Grunberg, and Marc A. Stern. 1999. Global Public Goods: International Cooperation in the 21st Century. New York: Oxford University Press.

Keohane, Robert. 1984. After Hegemony: Cooperation and Discord in the World Political Economy. Princeton: Princeton University Press.

Lancaster, Carol. 2007. Foreign Aid: Diplomacy, Development, Domestic Politics. Chicago: University of Chicago Press.

March, James G., and Johan P. Olsen. 1998. "The Institutional Dynamics of International Political Orders." International Organization 52 (4): 943-969.

Milner, Helen V. 1997. Interests, Institutions, and Information: Domestic Politics and International Relations. Princeton: Princeton University Press.

Milner, Helen V. 2006. "Why Multilateralism? Foreign Aid and Domestic Principal-Agent Problems." In Delegation and Agency in International Organizations, ed. Darren G. Hawkins, David A. Lake, Daniel L. Nielson, and Michael J. Tierney. New York: Cambridge University Press.

Mitchell, Ronald B., and Patricia M. Keilbach. 2001. "Situation Structure and Institutional Design: Reciprocity, Coercion, and Exchange." International Organization 55 (4): 891-917.

Myerson, Roger B. 1979. "Incentive Compatibility and the Bargaining Problem." Econometrica 47 (1): $61-74$. 
Nash, John F. 1950. “The Bargaining Problem.” Econometrica 18 (2): 155-162.

Nielson, Daniel L., and Michael J. Tierney. 2003. "Delegation to International Organizations: Agency Theory and World Bank Environmental Reform." International Organization 57 (2): 241-276.

Nordhaus, William D. 2010. "Economic Aspects of Global Warming in a Post-Copenhagen Environment." Proceedings of the National Academy of Sciences 107 (26): 11721 -11726.

Parson, Edward A. 2003. Protecting the Ozone Layer: Science and Strategy. New York: Oxford University Press.

Pedersen, Karl Rodolf. 2001. "The Samaritan's Dilemma and the Effectiveness of Development Aid." International Tax and Public Finance 8 (5-6): Pedersen2001.

Putnam, Robert D. 1988. "Diplomacy and Domestic Politics: The Logic of Two-Level Games." International Organization 42 (3): 427-460.

Rodrik, Dani. 1995. “Why Is There Multilateral Lending?” NBER Working Paper 5160.

Sandler, Todd. 2004. Global Collective Action. New York: Cambridge University Press.

Sandler, Todd, and Keith Hartley. 2001. "Economics of Alliances: The Lessons for Collective Action." Journal of Economic Literature 39 (3): 869-896.

Schelling, Thomas C. 1960. The Strategy of Conflict. Cambridge: Harvard University Press.

Sprinz, Detlef, and Tapani Vaahtoranta. 1994. "The Interest-Based Explanation of International Environmental Policy." International Organization 48 (1): 77-105.

Stewart, Richard B., Benedict Kingsbury, and Bryce Rudyk, eds. 2009. Climate Finance: Regulatory and Funding Strategies for Climate Change and Global Development. New York: New York University Press.

Stiglitz, Joseph E., and Scott J. Wallsten. 1999. "Public-Private Technology Partnerships: Promises and Pitfalls." American Behavioral Scientist 43 (1): 52-73.

Torsvik, Gaute. 2005. "Foreign Economic Aid; Should Donors Cooperate?" Journal of Development Economics 77 (2): 503-515.

Tsebelis, George. 2002. Veto Players: How Political Institutions Work. Princeton: Princeton University Press.

Victor, David G. 2011. Global Warming Gridlock: Creating More Effective Strategies for Protecting the Planet. New York: Cambridge University Press. 


\begin{tabular}{cccc}
$\frac{1}{1-p_{B}}$ & 1 & $t^{*}=D^{B}$ & \\
\hline$p_{B}$ & 1 & $\frac{\left(1-p_{B}\right) p_{A}+\left(1-p_{A}\right) p_{B}}{1-p_{A}}$
\end{tabular}

(a) Country $B$ faces strong domestic constraints with $p_{B} \geq \frac{1}{2}$, i.e., $\frac{1-p_{B}}{p_{B}} \leq 1$. Therefore, MFP formation is impossible regardless of $\frac{D^{A}}{D^{B}}$. If $\frac{D^{A}}{D^{B}}$ is smaller than $\frac{\left(1-p_{B}\right) p_{A}+\left(1-p_{A}\right) p_{B}}{1-p_{A}}$, the recipient chooses the low offer $t^{*}=D^{B}$. Otherwise it chooses the intermediate offer $t^{*}=D^{A}$.

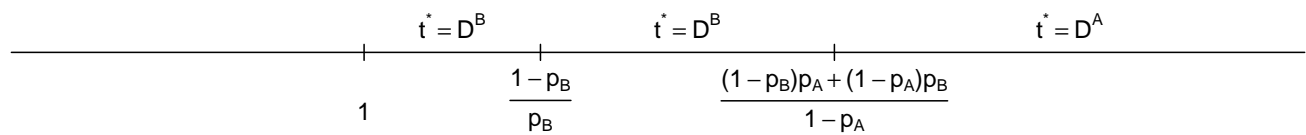

(b) Country $A$ faces strong domestic constraints with $p_{A}>\frac{1-p_{B}\left(1-p_{B}\right)}{1-2 p_{B}^{2}}$, i.e., $\frac{1-p_{B}}{p_{B}}<\frac{\left(1-p_{B}\right) p_{A}+\left(1-p_{A}\right) p_{B}}{1-p_{A}}$. Therefore, MFP formation is impossible regardless of $\frac{D^{A}}{D^{B}}$. If $\frac{D^{A}}{D^{B}}$ is smaller than $\frac{\left(1-p_{B}\right) p_{A}+\left(1-p_{A}\right) p_{B}}{1-p_{A}}$, the recipient chooses the low offer $t^{*}=D^{B}$. Otherwise it chooses the intermediate offer $t^{*}=D^{A}$.

$\begin{array}{ccc}t^{*}=D^{B} & t^{*}=D^{A}+D^{B} & t^{*}=D^{A} \\ 1 & \frac{\left(1-p_{B}\right) p_{A}+\left(1-p_{A}\right) p_{B}}{1-p_{A}} & \frac{1-p_{B}}{p_{B}}\end{array}$

(c) Countries $A$ and $B$ do not face strong domestic constraints as $p_{A}<\frac{1-p_{B}\left(1-p_{B}\right)}{1-2 p_{B}^{2}}$, i.e., $\frac{1-p_{B}}{p_{B}}>$ $\frac{\left(1-p_{B}\right) p_{A}+\left(1-p_{A}\right) p_{B}}{1-p_{A}}$. Therefore, MFP formation is possible for intermediate values of $\frac{D^{A}}{D^{B}}$. The recipient's offer is low $t^{*}=D^{B}$ if $\frac{D^{A}}{D^{B}}$ is smaller than $\frac{\left(1-p_{B}\right) p_{A}+\left(1-p_{A}\right) p_{B}}{1-p_{A}}$; medium $t^{*}=D^{A}$ if $\frac{D^{A}}{D^{B}}$ exceeds $\frac{1-p_{B}}{p_{B}}$, and high $t^{*}=D^{A}+D^{B}$ between these two intervals.

Figure 1: Illustration of Propositions 1, 2, and 3. Each bar graph shows the equilibrium offer (low $t^{*}=D^{B}$, medium $t^{*}=D^{A}$, or high $t^{*}=D^{A}+D^{B}$ ) as a function of the donor valuation ratio $\frac{D^{A}}{D^{B}}$. In the upper and middle bar graph, MFP formation is not possible because one of the donors is politically constrained with a high probability. In the lower bar graph, MFP formation is possible for intermediate values of $\frac{D^{A}}{D^{B}}$ because neither donor is constrained with a high probability. In other words, MFP formation depends on a high reward-risk ratio for gambling on the absence of political constraints in both countries. 